\title{
Variability of L Dwarfs in the Near Infrared
}

\author{
José A. Caballero ${ }^{1}$, Víctor J. S. Béjar ${ }^{1}$, Rafael Rebolo ${ }^{1,2}$ \\ ${ }^{1}$ Instituto de Astrofísica de Canarias, 38205, La Laguna, Tenerife, \\ Spain \\ ${ }^{2}$ Consejo Superior de Investigaciones Científicas, Spain
}

\begin{abstract}
We have obtained series of images in the near infrared $J$ and $K_{\mathrm{s}}$ bands for seven L-type dwarfs with a duration of 3 to 6 hours. We present results on: 1) the amplitude of variability associated with atmospheric changes over time scales from minutes to several hours; 2) the search for cool companions in wide orbits; 3 ) the search for transits of brown dwarfs and planetary companions in very close orbits.
\end{abstract}

\section{Observations and results}

The photometric variability of $\mathrm{L}$ and $\mathrm{T}$ dwarfs has been subject of study in a few recent studies (Bailer-Jones \& Mundt 1999, 2001; Terndrup et al. 1999; Clarke, Tinney \& Covey 2002), mostly performed in the optical region. Here, we present results on the photometric monitoring in the near IR $J$ and $K_{\mathrm{s}}$ bands for seven early to mid L-type field dwarfs selected from the literature (Kirkpatrick et al. $1999,2000)$.

The objects were observed on different nights using the infrared camera at the $1.52 \mathrm{~m}$ Telescopio Carlos Sánchez (Teide Observatory). We have performed differential aperture photometry over each pair of temporal series of the seven $\mathrm{L}$ dwarfs in both filters. An aperture of 1 FWHM was adopted to minimize photometric errors. Differential magnitudes have been obtained using the same method as that of Bailer-Jones \& Mundt. The reference level has been defined using stars in a field of $3^{\prime} \times 3^{\prime}$. Variable stars were discarded. A similar method to that in Martín et al. (2001) has been applied to quantify the deviation of the variability on our targets, comparing the standard deviation $(\sigma)$ of the target time series with respect to that of reference stars of similar magnitude. Also, a periodogram analysis have been performed, with negative results.

We have combined our series of observations to produce deep images in both the $J$ and $K_{\mathrm{s}}$ filters. Aperture (5 FWHM) and PSF photometry have been obtained for all the detected objects. Using $J-K_{\mathrm{s}}$ colors and negative detections in the Red plate of the Digital Sky Survey-2, we restrict the presence of faint late L- and T-spectral type companions to the L-dwarf primaries at wide separations. We have used absolute magnitude relations from Dahn et al. (2002), and distances and apparent magnitudes from the literature. 
Table 1. Shortened IAU name for the L dwarfs in our sample, spectral type, upper limits on the variability in both the $J$ and $K_{\mathrm{s}}$ filters, coolest spectral type of the faintest detectable companion, and the physical region searched around the L dwarf $\left(\sim 4^{\prime \prime}\right.$ to $\left.80^{\prime \prime}\right)$.

\begin{tabular}{cccccc}
\hline $\begin{array}{c}\text { Shortened } \\
\text { name }\end{array}$ & $\begin{array}{c}\text { Primary } \\
\text { Sp.T. }\end{array}$ & $\begin{array}{c}\sigma_{J} \\
(\mathrm{mmag})\end{array}$ & $\begin{array}{c}\sigma_{K_{\mathrm{s}}} \\
(\mathrm{mmag})\end{array}$ & $\begin{array}{c}\text { Secondary } \\
\text { Sp.T. }\end{array}$ & $\begin{array}{c}\text { Dist. range } \\
(\mathrm{AU})\end{array}$ \\
\hline 2M0015+35 & L2 & 30 & 37 & $>\mathrm{T} 6$ & $100-1000$ \\
$2 \mathrm{M} 0036+18$ & L3.5 & 16 & 25 & $>\mathrm{T} 8$ & $40-350$ \\
2M0058-06 & L0 & 24 & 38 & $>\mathrm{T} 3$ & $160-1000$ \\
2M0746+20 & L0.5 & 30 & 25 & $>\mathrm{T} 8$ & $50-1000$ \\
GJ 417B & L4.5 & 28 & 47 & $>\mathrm{T} 7$ & $130-430$ \\
2M1146+22 & L3 & 25 & 33 & $>\mathrm{T} 5$ & $70-1100$ \\
2M2224-01 & L2 & 14 & 11 & $>\mathrm{T} 8$ & $70-630$ \\
\hline
\end{tabular}

\section{Conclusions}

The combination of all the $J K_{\mathrm{s}}$ images for the seven $\mathrm{L}$ dwarfs resulted in final images with completeness magnitudes of $J \sim 18.0-19.0$ and $K_{\mathrm{s}} \sim 16.5-17.0$. Our search for cool companions at wide separations $(\sim 100-1000 \mathrm{AU})$ in these final images did not reveal any $\mathrm{T}$ dwarfs with spectral types earlier than those presented in the fifth column of Table 1.

Only one object, $2 \mathrm{M} 0015+35$, seems to be variable on a timescale of hours. For the rest, we can set upper limits on the variability in the range of $10-$ 40 mmag. The upper limits are similar to the variability levels found in the optical for similar objects.

We have not found evidence for eclipses due to close companions and conclude that there are no companions to our $\mathrm{L}$ dwarfs with radii greater than $\sim 0.3 \mathrm{R}_{\text {bd }}$ (slightly smaller than Neptune) at separations closer than $\sim 7 \mathrm{R}_{\text {bd }}$ $(\sim 0.3 \mathrm{AU})$ and orbital inclination angles greater than $82^{\circ}$.

\section{References}

Bailer-Jones, C. A. L \& Mundt, R. 1999, A\&A, 348, 800

Bailer-Jones, C. A. L \& Mundt, R. 2001, A\&A, 367, 218

Clarke, F. J., Tinney, C. G. \& Covey, K. R 2002, MNRAS, 332, 361

Dahn, C. C et al. 2002, AJ, accepted (astro-ph/0205050)

Kirkpatrick, J. D. et al. 1999, ApJ, 519, 802

Kirkpatrick, J. D. et al. 2000, AJ, 120, 447

Martín, E. L., Zapatero Osorio, M. R. \& Lehto, H. J. 2001, ApJ, 557, 822

Terndrup, D. M. et al. 1999, AJ, 118, 1814 